\title{
MicroRNA-424 expression predicts tumor recurrence in patients with hepatocellular carcinoma following liver transplantation
}

\author{
LIMING WU ${ }^{1-3}$, FEIBIAO YANG ${ }^{3,4}$, BINGYI LIN $^{2,3}$, XINHUA CHEN $^{2,3}$, SHENGYONG YIN $^{1,2}$, \\ FENG ZHANG $^{1,2}$, HAIYANG XIE ${ }^{1,2}$, LIN ZHOU $^{1,2}$ and SHUSEN ZHENG ${ }^{1-3}$
}

\begin{abstract}
${ }^{1}$ Department of Hepatobiliary and Pancreatic Surgery, The First Affiliated Hospital, Zhejiang University School of Medicine, Hangzhou 310003; ${ }^{2}$ Collaborative Innovation Center for Diagnosis and Treatment of Infectious Diseases;

${ }^{3}$ Key Lab of Combined Multi-Organ Transplantation, Ministry of Public Health; ${ }^{4}$ Department of Hepatobiliary Surgery, Yinzhou People's Hospital of Ningbo City, Ningbo, Zhejiang 315100, P.R. China
\end{abstract}

Received December 25, 2015; Accepted October 20, 2017

DOI: $10.3892 / \mathrm{ol} .2018 .8539$

\begin{abstract}
MicroRNA-424 (miR-424) has previously been described as a biomarker of poor prognosis in patients with hepatocellular carcinoma (HCC). In the present study, the clinical significance of miR-424 expression in predicting the rate of tumor recurrence in patients with $\mathrm{HCC}$ following liver transplantation (LT) was evaluated. miR-424 expression in HCC samples from 121 patients undergoing LT was examined, and the associations between clinical parameters and patient tumor recurrence were evaluated. The miR-424 expression level in cancer tissues was low compared with that in adjacent noncancerous tissues. Multivariate analyses revealed that low miR-424 expression was an independent prognostic factor for tumor recurrence in patients with HCC following liver transplantation. Patients who no longer met the Milan criteria and had decreased miR-424 expression levels exhibited earlier tumor recurrence following LT. In addition, the upregulation of miR-424 expression significantly reduced the migration, invasion and proliferation of HCC cells. Similarly, the downregulation of miR-424 in HCC cells significantly promoted the migration, invasion and proliferation of HCC cells.
\end{abstract}

\section{Introduction}

Hepatocellular carcinoma (HCC) is one of the most common types of malignant neoplasms worldwide, causing $\sim 700,000$ mortalities annually (1). Overall, half of these cases and mortalities were identified to occur in China, and were attributed to the high prevalence of hepatitis B virus (HBV)

Correspondence to: Dr Shusen Zheng, Department of Hepatobiliary and Pancreatic Surgery, The First Affiliated Hospital, Zhejiang University School of Medicine, 79 Qingchun Road, Hangzhou 310003, P.R. China

E-mail: shusenzheng@zju.edu.cn

Key words: microRNA-424, hepatocellular carcinoma, liver transplantation infections and liver cirrhosis $(2,3)$. As a potentially curative treatment for liver cirrhosis and cancer, liver transplantation (LT) is widely used to treat selected patients with early-stage HCC, and it has the greatest benefit among all the established therapeutic options $(4,5)$. However, the frequent recurrence and high metastasis rates of HCC hinders positive outcomes for patients. Due to the multiple genetic alterations that are responsible for the progression of HCC (6), exploration of the molecular mechanism of HCC is important for disease diagnosis, treatment and prediction of outcomes.

MicroRNAs (miRNAs/miR) are a class of small, non-coding RNA molecules (containing 22 nucleotides) identified in plants, animals and certain viruses. They function in RNA silencing and the post-transcriptional regulation of gene expression $(7,8)$. Increasing evidence supports a marked association between cancer and miRNAs (9). It is well documented that aberrant miRNA expression serves a significant role in carcinogenesis and cancer development (10). miRNAs may regulate the biological behaviors of cancer, including proliferation, apoptosis and invasion. Therefore, exploration of miRNAs may assist in identifying novel diagnostic or prognostic markers and treatment targets.

The Milan criteria (11) for identifying candidate patients with HCC for LT have been accepted worldwide. However, they ignore the molecular biomarker expression patterns in HCC and have been criticized for being too restrictive (12). Our prior studies aimed to explore more inclusive criteria for identifying LT candidates within the patients with HCC population $(12,13)$. The noncoding RNA molecules are potentially valuable markers that may assist in predicting HCC recurrence following LT, their aberrant expression was significantly associated with increased risk of recurrence (14). MicroRNA-424 (miR-424) was previously demonstrated to inhibit the proliferation and metastasis of $\operatorname{HCC}(15,16)$. In the present study, the expression pattern of miR-424 in HCC tissues from patients who received transplant therapy, and its association with clinicopathological parameters were investigated, and the utility of miR-424 in predicting tumor recurrence in patients with HCC following LT was explored. The functional implications of miR-424 in HCC were also explored using in vitro and in vivo assays. 


\section{Materials and methods}

Patient samples. A total of 121 patients with HCC, who were treated with LT from January 2003 to December 2010 at The First Affiliated Hospital, Zhejiang University School of Medicine (Zhejiang, China), were enrolled in the present study. The inclusion criteria were as previously described (17). Ages of patients ranged from 41 to 73 years old and the male-female ratio was 112/9. All patients were HBV-surface antigen-positive $\left(\mathrm{HBsAg}^{+}\right)$and none were hepatitis $\mathrm{C}$ virus (HCV)-positive. The present study was approved by the Medical Ethics Committee of The First Affiliated Hospital of Zhejiang University, and written informed consent was obtained from all patients. The patients were diagnosed with HCC either prior or subsequent to transplantation via histopathological diagnosis. The complete clinical and laboratory data were available prior to surgery and during follow-up. The follow-up course and diagnostic criteria of recurrence have been previously described (18). The distribution of clinicopathological data in the study cohort is summarized in Table I. Specimens of cancer/noncancerous tissues and clinical information were available from these patients subsequent to obtaining written informed consent.

Postoperative follow-up. The surviving patients were followed up at the outpatient clinic. The median follow-up duration was 25.12 months. Tumor recurrence was monitored by the $\alpha$-fetoprotein (AFP) levels, ultrasonography, chest X-ray and emission computed tomography every 3 months for the first 2 years, and semiannually thereafter. Imaging techniques, either intrahepatically or extrahepatically (lymph nodes and distant metastases) were used to diagnose recurrence. However, a simple increase in the AFP did not conclusively indicate recurrence.

Cell lines and chemical reagents. The normal liver cell line, LO2; liver cancer cell lines, SMMC-7721, Huh-7, HepG2, Bel-7402, and SK-HEP-1; and metastatic human HCC cell line, HCCLM3, were purchased from the American Type Culture Collection (Manassas, VA, USA), the Shanghai Institute of Cell Biology (Shanghai, China) and the Liver Cancer Institute of Fudan University (Shanghai, China). LO2, SMMC-7721 and BEL-7402 cells were cultured in RMPI 1640 (Biological Industries, Kibbutz Beit-Haemek, Israel); Huh-7, HepG2, SK-HEP-1 and HCCLM3 cells were maintained in Dulbecco's modified eagle's medium (DMEM; Biological Industries). All cells were incubated at $37^{\circ} \mathrm{C}$ in a humidified environment containing $5 \% \mathrm{CO}_{2}$.

Reverse transcription-quantitative polymerase chain reaction $(R T-q P C R)$ analysis. TRIzol ${ }^{\circledR}$ (Invitrogen; Thermo Fisher Scientific, Inc., Waltham, MA, USA) and miScript II RT kits (Qiagen, Inc., Valencia, CA, USA) were used to extract RNA from LO2, SMMC-7721, Huh-7, HepG2, Bel-7402 and SK-HEP-1 cell lines. Following incubation for $60 \mathrm{~min}$ at $37^{\circ} \mathrm{C}$, the reverse transcription mix was placed in a water bath at $95^{\circ} \mathrm{C}$ and then transferred to refrigerator at $4^{\circ} \mathrm{C}$. qPCR was conducted using the miScript SYBR-Green kit (Qiagen, Inc.) on an Applied Biosystems ABI 7700 System (Thermo Fisher Scientific, Inc.), according to the manufacturer's protocol.
miR-424 primers were purchased from Qiagen, Inc. GAPDH was used as internal control, with sequences as follows: Sense, AGGTCGGAGTCAACGGATTTG and anti-sense, GTGATG GCATGGACTGTGGT59. For miR-424 detection, U6 snRNA was used as the endogenous control. Each experiment was performed in three replicates. The $2^{-\Delta \Delta \mathrm{Cq}}$ method was used to analyze the PCR data (19).

Cell model construction and infection. SK-HEP-1 (highest miR-424 expression) and Huh-7 cells (last but one low miR-424 expression, selected as they were more suitable for tumorgenicity) were infected with lentiviral vectors carrying a miRNA mimic/inhibitor or nonsense controls (NC2) (GenePharma Co., Shanghai, China) with polybrene, according to the manufacturer's protocol. The sequences were as follows: miR-424 inhibitor-1, 5' CAGCAGCAATTCATG TTTTGAA3'; miR-424 inhibitor-2, 5' TTCAAAACATGA ATTGCTGCTG3'; NC2, 5'TTCTCCGAACGTGTCACGT3'.

Analysis of cell viability, migration and invasion. Cell viability of the aforementioned transfected HCC cells (SK-HEP-1 and Huh-7) was determined using the CCK-8 assay (Dojindo Molecular Technologies, Inc., Kumamoto, Japan), according to the manufacturer's protocol. Cells $\left(5 \times 10^{3}\right)$ were seeded into the wells of a 96 -well plate. Subsequent to the cells being incubated at $37^{\circ} \mathrm{C}$ for different time periods (24, 48, 72 and $96 \mathrm{~h})$, DMEM medium was replaced with $100 \mu \mathrm{l}$ DMEM medium supplemented with $10 \mu \mathrm{lCCK}-8$ reagent. The cells were incubated for $2 \mathrm{~h}$ at $37^{\circ} \mathrm{C}$. Finally, the optical density was measured at $450 \mathrm{~nm}$.

Transwell invasion and migration experiments were conducted as previously described (20). Briefly, the polycarbonate membrane of the upper chamber was coated with Matrigel for invasion experiments. A total of $72 \mathrm{~h}$ after incubation, SK-HEP-1 and Huh-7 cells with corresponding virus transfection were collected for Transwell experiments. Cells $(2 \times 104)$ were seeded into the upper chambers, which contained DMEM medium without fetal bovine serum (Biological Industries). DMEM medium with 15\% FBS was added to the lower compartments. After $24 \mathrm{~h}$ (for migration experiments) or $48 \mathrm{~h}$ (for invasion experiments), the cells on the lower surface were fixed in $95 \%$ methanol for $10 \mathrm{~min}$ at room temperature and stained for $20 \mathrm{~min}$ with $0.1 \%$ crystal violet at room temperature. The cells were counted and images were captured using digital microscopy (magnification, x100). A total of 3 fields per filter were counted in each group. The experiments were performed in triplicate.

Xenograft model analysis. To investigate the anti-proliferative effect of miR-424 on HCC cells in vivo, a model with nude mice bearing HCC cell xenografts was established: A total of fourteen five-week-old male athymic nude mice with a median weight of $14 \mathrm{~g}$ (Vital River Laboratory Animal Technology Co., Ltd., Beijing, China) were maintained under pathogen-free conditions and were provided with ad libitum access to sterilized food and water at $26-28^{\circ} \mathrm{C}$ and were exposed to light for $12 \mathrm{~h}$ every day. Huh-7 cells $\left(5 \times 10^{6}\right)$ were infected with a miR-424 mimic or nonsense control, injected subcutaneously into the right armpit, near the chest, of each nude mouse. Seven mice were used in each group. Six weeks 
Table I. Association between miR-424 expression and the clinicopathological features of patients with human hepatocellular carcinoma.

Tumor miR-424 expression

Variables Low High P-value ${ }^{\mathrm{a}}$

Age, years

$\leq 50$

30

$>50$

31

Sex

Male

55

6

Portal vein tumor thrombi

Absent

39

22

Present

$>400$

33

$>5$

21

40

Single
33

27

57

3

41

40

19

29

31

33

27

20

30

30

miR, microRNA. Patients with HCC receiving liver transplantation were segregated into miR-424-high/low expression groups (calculation of

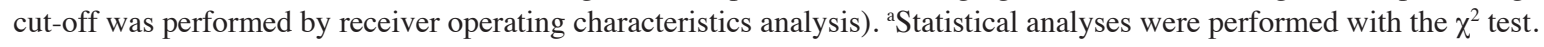

following the injection, the mice were sacrificed by removal of the cervical spine. The maximum tumor volume obtained in the mice was $1.8 \mathrm{~cm}^{3}$. Tumor volume $=1 / 2\left(\right.$ length $\mathrm{x}$ width $\left.{ }^{2}\right)$.

Statistical analysis. The experimental data are presented as the mean \pm standard deviation or frequency. Continuous data were analyzed using unpaired Student's t-test to assess differences between the two groups. Categorical data were analyzed using the $\chi^{2}$ test. The recurrence-free survival probability was analyzed with the Kaplan-Meier method, and a log-rank test was used to estimate the differences between groups. Independent prognostic indicators were determined in the multivariate analysis using Cox's proportional hazards model. The expression correlation was analyzed by a two-tailed linear Spearman's rank test. All statistical analyses were performed using SPSS v.16.0 (SPSS, Inc., Chicago, IL, USA) and GraphPad Prism 5.0 (GraphPad Software, La Jolla, CA, USA). P<0.05 was considered to indicate a statistically significant difference.

\section{Results}

miR-424 expression in liver cancer cell lines and the tissues of patients with HCC. The expression levels of miR-424 in a human normal liver cell line, LO2, and six liver cancer cell lines were measured using RT-qPCR. Of the 6 liver cancer cell lines, 5 (HCC-LM3, Huh-7, SMMC-7721, HepG2 and BEL-7402) expressed lower levels of miR-424 compared with the normal liver cell line (Fig. 1A). Additionally, the relative expression levels of miR-424 in tumors and adjacent non-tumorous tissues from 121 patients with HCC were measured. The tumors expressed significantly lower levels of miR-424 compared with the adjacent non-tumorous tissues. Furthermore, recurrent tumors expressed lower miR-424 levels compared with non-recurrent tumors ( $\mathrm{P}<0.01$; Fig. 1B).

Low mir-424 expression is associated with early tumor recurrence in patients with HCC following LT. To explore the utility of miR-424 as a promising molecular marker for predicting the prognosis of patients with HCC, the recurrence-free survival times between 121 patients with HCC who expressed high or low expression levels of mir-424 were compared, based on extensive clinical follow-up data. Firstly, mir-424 expression did not correlate with any, single clinicopathological characteristic in the 121 patients, including the age, sex, portal vein tumor thrombi, AFP, histopathological grade, tumor size or tumor number, when stratified by expression level (high or low) (Table I). Furthermore, the univariate recurrence-free survival analysis demonstrated 
Table II. Univariate analyses of predictors of recurrence in patients with hepatocellular carcinoma following liver transplantation.

\begin{tabular}{|c|c|c|c|}
\hline \multirow[b]{2}{*}{ Variables } & \multicolumn{2}{|c|}{ Tumor recurrence } & \multirow[b]{2}{*}{ P-value } \\
\hline & Negative & Positive & \\
\hline \multicolumn{4}{|l|}{ Tumor size, $\mathrm{cm}$} \\
\hline$\leq 5$ & 45 & 25 & \multirow[t]{2}{*}{$<0.001$} \\
\hline$>5$ & 8 & 43 & \\
\hline \multicolumn{4}{|c|}{ Preoperative $\alpha$-fetoprotein level, ng/ml } \\
\hline$\leq 400$ & 32 & 24 & \multirow[t]{2}{*}{0.009} \\
\hline$>400$ & 21 & 44 & \\
\hline \multicolumn{4}{|c|}{ Portal vein tumor thrombi } \\
\hline Absent & 44 & 36 & \multirow[t]{2}{*}{$<0.001$} \\
\hline Present & 9 & 32 & \\
\hline \multicolumn{4}{|l|}{ Milan criteria ${ }^{\mathrm{b}}$} \\
\hline Within criteria & 30 & 11 & \multirow[t]{2}{*}{$<0.001$} \\
\hline Beyond criteria & 12 & 68 & \\
\hline \multicolumn{4}{|c|}{ miR-424 expression } \\
\hline Low & 34 & 7 & \multirow[t]{2}{*}{$<0.001$} \\
\hline High & 19 & 61 & \\
\hline \multicolumn{4}{|c|}{ Meeting Milan criteria, miR-424 expression } \\
\hline Low & 15 & 3 & \multirow[t]{2}{*}{0.669} \\
\hline High & 19 & 4 & \\
\hline \multicolumn{4}{|c|}{ No longer meeting Milan criteria, miR-424 expression } \\
\hline Low & 8 & 35 & \multirow[t]{2}{*}{0.012} \\
\hline High & 11 & 26 & \\
\hline
\end{tabular}

${ }^{a}$ Statistical analyses were conducted using the Kaplan-Meier method (log-rank test). ${ }^{\text {b } I n ~ t h e ~ a b s e n c e ~ o f ~ m a c r o v a s c u l a r ~ i n v a s i o n, ~ M i l a n ~ c r i t e r i a ~}$ are met if a single tumor is $\leq 5 \mathrm{~cm}$ in diameter, or if $\leq 3$ tumor nodules, each $\leq 3 \mathrm{~cm}$ in diameter, are present; miR, microRNA.

Table III. microRNA-424 expression in hepatocellular carcinoma is an independent predictive factor for recurrence in patients following liver transplantation.

Cumulative recurrence

\begin{tabular}{lc} 
Variables & Hazard ratio (95\% CI) $^{\text {P-value }^{\mathrm{a}}}$ \\
\hline $\begin{array}{l}\text { Milan criteria } \\
\text { No longer meeting vs. meeting }\end{array}$ & $3.743(1.439-9.738)$ \\
$\begin{array}{l}\text { Tumor size, cm } \\
>5 \text { vs. } \leq 5\end{array}$ & $2.158(1.205-3.864)$ \\
$\begin{array}{l}\text { microRNA-424 expression } \\
\text { High vs. low }\end{array}$ & 0.007 \\
\hline
\end{tabular}

${ }^{\mathrm{a} C o x}$ 's proportional hazards regression; CI, confidence interval.

that the tumor size, preoperative AFP level, PPTV, Milan criteria (11) and mir-424 expression serve as risk factors for tumor recurrence following LT (Table II). In addition, COX multivariate analysis revealed that for patients who no longer met the Milan criteria, a tumor size of $>5 \mathrm{~cm}$ and high mir-424 expression levels were independent risk factors for tumor recurrence following LT (Table III).
The overexpression of miR-424 predicted earlier recurrence times in patients with $\mathrm{HCC}$ who underwent LT $(\mathrm{P}=0.029$; Fig. 1C). The results of the present study revealed that the Milan criteria effectively predicted the risk of tumor recurrence [Table III; no longer meeting vs. meeting, hazard ratio $(\mathrm{HR})=3.743 ; \mathrm{P}=0.007]$ in patients following $\mathrm{LT}$, which is consistent with previous studies (16). Notably, in patients 

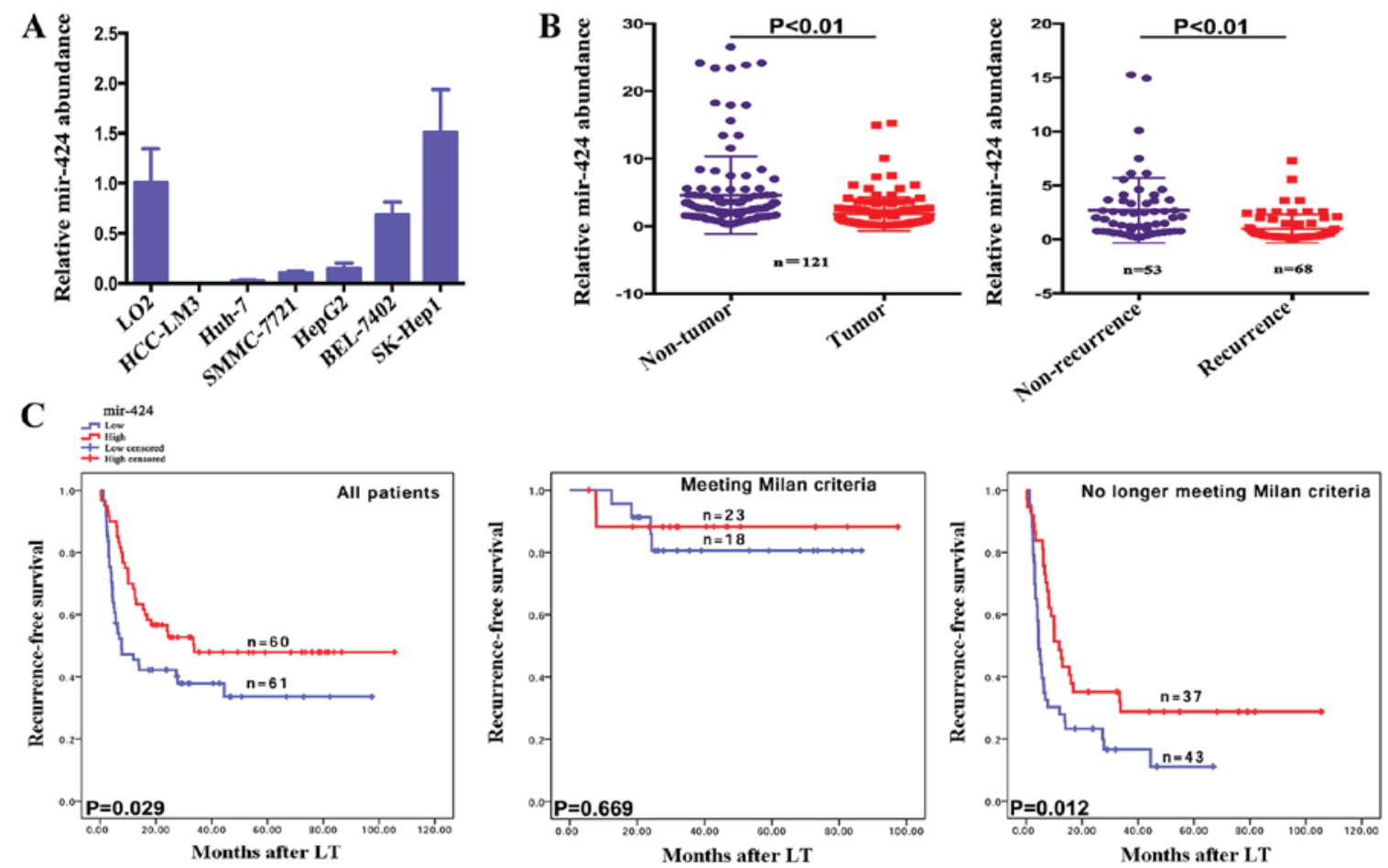

Figure 1. miR-424 expression and its clinical significance. (A) Relative expression levels of miR-424 in the normal liver and in liver cancer cell lines. (B) Relative expression levels of miR-424 in tumors and adjacent non-tumorous tissues or in recurrent and non-recurrent tumors from 121 patients with HCC. (C) Comparisons of recurrence-free survival rates according to the expression of miR-424 in tumors from patients with HCC. The left graph includes all patients. The middle graph includes patients meeting the Milan criteria, and the right graph includes patients who no longer meet the Milan criteria. miR, microRNA; LT, liver transplantation; HCC, hepatocellular carcinoma.

who met the Milan criteria, additional classification based on miR-424 expression did not result in significant differences in the recurrence-free survival times ( $\mathrm{P}=0.669$; Fig. 1C). However, patients who no longer met the Milan criteria and who exhibited increased miR-424 expression levels experienced earlier tumor recurrence following LT ( $\mathrm{P}=0.012$, Fig. 1C). Taken together, these clinical data demonstrate that miR-424 expression is an important indicator for predicting tumor recurrence in patients with HCC following LT ( $\mathrm{P}=0.029)$, which may also be valid in patients who no longer meet the Milan criteria $(\mathrm{P}=0.012)$.

Effects of miR-424 expression on the proliferation, migration and invasiveness of HCC cells. SK-HEP-1 and Huh-7 cells were treated with an miR-424 mimic or miR-424 inhibitor. Consequently, miR-424 expression was effectively upregulated or downregulated (Fig. 2A). The Transwell assay, CCK-8 assay and an HCC xenograft model demonstrated that the upregulation of miR-424 expression significantly reduced the migration, invasiveness (Fig. 2B), proliferation (Fig. 2C) of HCC cells, and also inhibited the tumor volume in nude mice (Fig. 2D). The downregulation of miR-424 in HCC cells significantly promoted the migration, invasion and proliferation of hepatocellular carcinoma cells (Fig. 2B and C).

\section{Discussion}

LT is a potentially curative treatment for HCC. Despite the development of diagnosis and treatment protocols for HCC in previous decades, the prognosis of HCC remains poor. HCC frequently recurs following surgery, which is primarily attributable to the presence of microscopic extrahepatic metastatic foci prior to LT (21). This recurrence remains the major obstacle for improving the long-term survival rates of patients with HCC (22).

The Milan criteria are frequently used in patient selection for LT. However, the Milan criteria that ignore the molecular biomarker expression patterns have been criticized for being too restrictive, and patients with tumors beyond the Milan criteria may also have a satisfactory prognosis (12). The present study considers the lack of biological molecular markers to be a limitation of the Milan criteria. Biological molecules that reflect tumor characteristics or immunity status are valuable for addition to the LT criteria for patients with HCC, which is recurrently evaluated at The First Affiliated Hospital, Zhejiang University School of Medicine (12-14,17).

The results of the present study revealed that in patients who are beyond the Milan criteria, those with increased miR-424 expression experienced a longer recurrence-free time. Patients with HCC beyond the Milan criteria, who exhibited a high miR-424 expression level in tumor tissues may be recommended as candidates for LT, thus potentially significantly increasing the number of patients with HCC who are eligible for LT. However, it appears too difficult to use a single molecular marker to efficiently diagnose $\mathrm{HCC}$ or predict recurrence $(23,24)$, which is also a limitation of the present study. However, the advance of high-throughput sequencing technology would promote the establishment of a diagnostic or prognostic model of multiple molecular biomarkers. Future studies may also evaluate additional molecular markers and patient samples to establish a prognostic model of recurrence and mortality in patients with HCC following LT, and explore improved prognostic criterion 

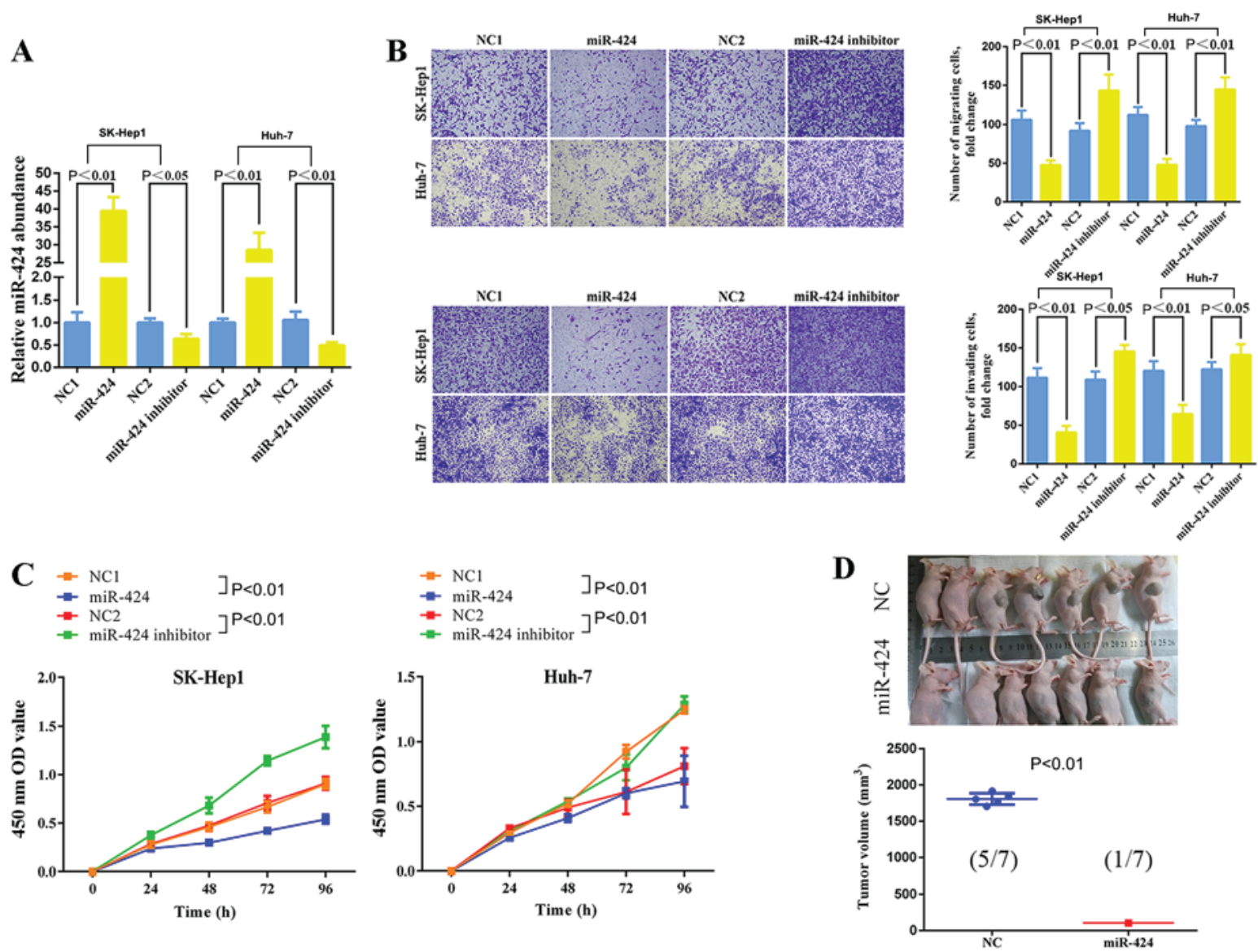

Figure 2. Effect of the miR-424 on the invasiveness and proliferation of SK-HEP-1 and Huh-7 cells. (A) Changes in miR-424 in HCC cells following miR-424 mimic or miR-424 inhibitor treatment for $48 \mathrm{~h}$. (B) Change in the migration (upper panel) and invasiveness (lower panel) of HCC cells following miR-424 mimic or miR-424 inhibitor treatment for $48 \mathrm{~h}$ (magnification, $\mathrm{x} 100$ ). The bars in the bar graph indicate the relative number of migrated or invaded cells per field (at magnification, x400). (C) Following miR-424 mimic or miR-424 inhibitor treatment for 24, 48, 72 and $96 \mathrm{~h}$, a CCK-8 assay was used to detect changes in cell viability. Huh-7 cells following miR-424 mimic or NC treatment were implanted subcutaneously into the flanks of nude mice. After 4 weeks, all the mice were sacrificed and the tumors volumes were measured. The maximum tumor volumes obtained in the mice was $1.8 \mathrm{~cm}^{3}$. Tumor volume $=1 / 2(\text { length } \mathrm{x} \text { width })^{2}$. $\mathrm{NC} 1$ was the negative control for the miR-424 mimic. NC2 was negative control for miR-424 inhibitor. Data are presented as the mean \pm standard deviation (t-test). miR, microRNA; NC, negative control; HCC, hepatocellular carcinoma.

for LT in patients with HCC. The present study, along with our previous studies $(14,17)$, provides valuable insight for the establishment of such a model. A preoperative detection of miR-424 expression in liver biopsy specimens and circulating blood cells should be performed in future studies.

Additionally, the biological role of miR-424 in HCC cell proliferation and invasion was explored with in vitro assays. Knockdown of miR-424 promoted the invasion and proliferation of the HCC cell lines SK-HEP-1 and Huh-7. Upregulation of miR-424 significantly reduced the invasive capability and suppressed the growth of HCC cells in vitro and in vivo. The results of the functional assays conducted in the present study are consistent with those of prior studies (15), and theoretically support the clinical data concerning the role of miR-424 in predicting the prognosis of patients with HCC following LT. However, the precise mechanisms of miR-424-induced HCC cell invasion and proliferation regulation remain unclear.

In conclusion, the present study presents miR-424 as a valuable biomarker for predicting recurrence in patients with HCC following LT. These results are important for establishing additional molecular markers that may be used in a prognostic model for recurrence and mortality in patients with HCC following LT. The limitation of the present study is that additional exploration is required to determine the exact mechanisms by which miR-424 regulates malignant biological behavior in HCC cells.

\section{Acknowledgements}

Not applicable.

\section{Funding}

The present study was supported by the Natural Science Foundation of Zhejiang Province (grant no. LY15H160021), the National Natural Science Foundation (grant nos. 81372425; 81572954), and the Foundation for Innovative Research Groups of the National Natural Science Foundation of China (grant no. 81421062).

\section{Availability of data and materials}

The datasets used and/or analyzed during the current study are available from the corresponding author on reasonable request. 


\section{Authors' contributions}

LW, LZ and SZ designed the study and wrote the manuscript. FY, BL and XC collected and analyzed the data. SY, HX and $\mathrm{FZ}$ performed the cell assays and animal experiments.

\section{Ethics approval and consent to participate}

The present study was approved by the Medical Ethics Committee of The First Affiliated Hospital of Zhejiang University, and written informed consent was obtained from all patients.

\section{Consent for publication}

Written informed consent was obtained from all patients.

\section{Competing interests}

The authors declare that they have no competing interests.

\section{References}

1. Jemal A, Bray F, Center MM, Ferlay J, Ward E and Forman D: Global cancer statistics. CA Cancer J Clin 61: 69-90, 2011.

2. El-Serag HB and Rudolph KL: Hepatocellular carcinoma: Epidemiology and molecularcarcinogenesis. Gastroenterology 132: 2557-2576, 2007

3. Ferlay J, Soerjomataram I, Dikshit R, Eser S, Mathers C, Rebelo M, Parkin DM, Forman D and Bray F: Cancer incidence and mortality worldwide: Sources, methods and major patterns in GLOBOCAN 2012. Int J Cancer 136: E359-E386, 2015.

4. Dutkowski P, Linecker M, DeOliveira ML, Mullhaupt B and Clavien PA: Challenges to liver transplantation and strategies to improve outcomes. Gastroenterology 148: 307-323, 2015.

5. Ramesh H: Resection for hepatocellular carcinoma. J Clin Exp Hepatol 4 (Suppl 3): S90-S96, 2014.

6. Arzumanyan A, Reis HM and Feitelson MA: Pathogenic mechanisms in HBV- and HCV-associated hepatocellular carcinoma. Nat Rev Cancer 13: 123-135, 2013.

7. Ambros V: The functions of animal microRNAs. Nature 431: 350-355, 2004

8. Bartel DP: MicroRNAs: Genomics, biogenesis, mechanism and function. Cell 116: 281-297, 2004

9. Catela Ivkovic T, Voss G, Cornella H and Ceder Y: microRNAs as cancer therapeutics: A step closer to clinical application. Cancer Lett 407: 113-122, 2017.

10. Hayes J, Peruzzi PP and Lawler S: microRNAs in cancer: Biomarkers, functions and therapy. Trends Mol Med 20: 460-469, 2014.

11. Mazzaferro V, Regalia E, Doci R, Andreola S, Pulvirenti A, Bozzetti F, Montalto F, Ammatuna M, Morabito A and Gennari L: Liver transplantation for the treatment of small hepatocellular carcinomas in patients with cirrhosis. N Engl J Med 334: 693-699, 1996.
12. Xu X, Lu D, Ling Q, Wei X, Wu J, Zhou L, Yan S, Wu L, Geng L, $\mathrm{Ke} \mathrm{Q}$, et al: Liver transplantation for hepatocellular carcinoma beyond the Milan criteria. Gut 65: 1035-1041, 2016.

13. Zheng SS, Xu X, Wu J, Chen J, Wang WL, Zhang M, Liang TB and Wu LM: Liver transplantation for hepatocellular carcinoma: Hangzhou experiences. Transplantation 85: 1726-1732, 2008.

14. Yang Z, Zhou L, Wu LM, Lai MC, Xie HY, Zhang F and Zheng SS: Overexpression of long non-coding RNA HOTAIR predicts tumor recurrence in hepatocellular carcinoma patients following liver transplantation. Ann Surg Oncol 18: 1243-1250, 2011.

15. Yang H, Zheng W, Shuai X, Chang RM, Yu L, Fang F and Yang LY: microRNA-424 inhibits Akt3/E2F3 axis and tumor growth in hepatocellular carcinoma. Oncotarget 6: 27736-27750, 2015.

16. Zhang Y, Li T, Guo P, Kang J, Wei Q, Jia X, Zhao W, Huai W, Qiu Y, Sun L and Han L: miR-424-5p reversed epithelial-mesenchymal transition of anchorage-independent HCC cells by directly targeting ICAT and suppressed HCC progression. Sci Rep 4: 6248, 2014.

17. Zhang F, Wu LM, Zhou L, Chen QX, Xie HY, Feng XW and Zheng SS: Predictive value of expression and promoter hypermethylation of XAF1 in hepatitis B virus-associated hepatocellular carcinoma treated with transplantation. Ann Surg Oncol 15: 3494-3502, 2008.

18. Wu LM, Zhang F, Xie HY, Xu X, Chen QX, Yin SY, Liu XC, Zhou L, Xu XB, Sun YL and Zheng SS: MMP2 promoter polymorphism (C-1306T) and risk of recurrence in patients with hepatocellular carcinoma after transplantation. Clin Genet 73: 273-278, 2008.

19. Livak KJ and Schmittgen TD: Analysis of relative gene expression data using real-time quantitative PCR and the 2(-Delta Delta C(T)) method. Methods 25: 402-408, 2001.

20. Lai MC, Yang Z, Zhou L, Zhu QQ, Xie HY, Zhang F, Wu LM, Chen LM and Zheng SS: Long non-coding RNA MALAT-1 overexpression predicts tumor recurrence of hepatocellular carcinoma after liver transplantation. Med Oncol 29: 1810-1816, 2012.

21. Kaiser J: The cancer stem cell gamble. Science 347: 226-229, 2015.

22. Zimmerman MA, Ghobrial RM, Tong MJ, Hiatt JR, Cameron AM, Hong J and Busuttil RW: Recurrence of hepatocellular carcinoma following liver transplantation: A review of preoperative and postoperative prognostic indicators. Arch Surg 143: 182-188, 2008.

23. Schutte K, Schulz C, Link A and Malfertheiner P: Current biomarkers for hepatocellular carcinoma: Surveillance, diagnosis and prediction of prognosis. World J Hepatol 7: 139-149, 2015.

24. Kim JH, Sohn BH, Lee HS, Kim SB, Yoo JE, Park YY, Jeong W, Lee SS, Park ES and Kaseb A: Genomic predictors for recurrence patterns of hepatocellular carcinoma: Model derivation and validation. PLoS Med 11: e1001770, 2014.

(i) $(-)$ This work is licensed under a Creative Commons Attribution-NonCommercial-NoDerivatives 4.0 International (CC BY-NC-ND 4.0) License. 